\title{
Progression free survival and overall survival of CDK 4/6 inhibitors plus endocrine therapy in metastatic breast cancer: a systematic review and meta-analysis
}

\author{
Michela Piezzo \\ Istituto Nazionale Tumori IRCCS Fondazione Pascale \\ Paolo Chiodini \\ Universita degli Studi della Campania Luigi Vanvitelli \\ Maria Riemma \\ Istituto Nazionale Tumori IRCCS Fondazione Pascale \\ Stefania Cocco \\ Istituto Nazionale Tumori IRCCS Fondazione Pascale \\ Roberta Caputo \\ Istituto Nazionale Tumori IRCCS Fondazione Pascale \\ Daniela Cianniello \\ Istituto Nazionale Tumori IRCCS Fondazione Pascale \\ Germira Di Gioia \\ Istituto Nazionale Tumori IRCCS Fondazione Pascale \\ Vincenzo Di Lauro \\ Istituto Nazionale Tumori IRCCS Fondazione Pascale \\ Francesca Di Rella \\ Istituto Nazionale Tumori IRCCS Fondazione Pascale \\ Giuseppina Fusco \\ Istituto Nazionale Tumori IRCCS Fondazione Pascale \\ Giovanni lodice \\ Istituto Nazionale Tumori IRCCS Fondazione Pascale

\section{Francesco Nuzzo} \\ Istituto Nazionale Tumori IRCCS Fondazione Pascale

\section{Carmen Pacilio} \\ Istituto Nazionale Tumori IRCCS Fondazione Pascale

\section{Matilde Pensabene} \\ Istituto Nazionale Tumori IRCCS Fondazione Pascale \\ Michelino De Laurentiis ( $\nabla$ delauren@breastunit.org ) \\ Istituto Nazionale Tumori IRCCS Fondazione Pascale https://orcid.org/0000-0001-9009-1572
}

\section{Research article}

Keywords: metastatic breast cancer, hormone therapy, CDK4/6 inhibitors, overall survival, subgroup analysis, hormone receptors

Posted Date: February 21st, 2020

DOI: https://doi.org/10.21203/rs.2.24219/v1 
License: @ (i) This work is licensed under a Creative Commons Attribution 4.0 International License. Read Full License

Version of Record: A version of this preprint was published at International Journal of Molecular Sciences on September 3rd, 2020. See the published version at https://doi.org/10.3390/ijms21176400. 


\section{Abstract}

PURPOSE: The introduction of CDK4/6 inhibitors plus endocrine therapy (ET) represents the most relevant advance in the management of HR-positive/HER2-negative metastatic breast cancer. We carried out a meta-analysis of randomized controlled trials (RCTs) with the aims of better characterising the efficacy of CDK4/6 inhibitors in some relevant subgroups and of testing heterogeneity between different compounds with particular focus on their ability to improve OS.

METHODS: We performed a systematic literature search to identify phase II/III RCTs of CDK4/6 inhibitors plus ET in Alsensitive and Al-resistant patients. Pooled estimates of HRs were computed for PFS, OS and ORR analysis, by using both a fixed and random effect model. Predefined subgroup analyses were performed to better understand treatment effect concerning specific patients' characteristics. Pooled survival curves were generated by pooling the data of all trials.

RESULTS: 8 RCTs were included. Adding a CDK4/6 inhibitors to ET is beneficial in terms of PFS irrespective of the presence or not of visceral metastases, the number of metastatic sites, and the length of the TFI. The addition of CDK4/6 inhibitors significantly improves OS in Al-sensitive (HR 0.75, 95\% Cl [0.63-0.89]) and Al-resistant patients (HR 0.77, 95\% Cl [0.67-0.89]). Pooled data from each single drug show that Palbociclib remains the only class member not showing a statistically significant HR for OS (HR 0.83, 95\% Cl [0.68-1.02]).

CONCLUSION: Our meta-analysis confirms the efficacy of CDK4/6 inhibitors overall and in major patients subgroups, supporting the use of CDK4/6 inhibitors plus ET as standard treatment for most HR+ MBC patients.

\section{Introduction}

Breast cancer (BC) is the most common cancer and the most common cause of cancer-related death in women and it represents, with 266.000 new cases estimated in 2018 in USA, about 30\% of all diagnosed tumours [1]. Despite the important advances in terms of prevention, diagnosis and availability of new treatments, metastatic breast cancer (MBC) remains an incurable disease and its evolution depends on several factors, such as site and extension of metastasis, histopathological characteristics and molecular profile of tumour.

In the last decade, preclinical and clinical research focused on identification of new treatment options able to prolong or restore the endocrine sensitivity, delaying the use of chemotherapy and improving the survival and the quality of life of these patients. In this scenario, deregulation of cell cycle represented one of the most interesting therapeutical targets, since dysregulation of cyclin D-CDK4/6-pRb pathway is frequent in hormone receptor positive (HR+) BC and represents a key mediator of endocrine resistance [2,3]. Targeting CDK4/6 resulted in an efficient inhibition of this pathway in $H R+B C[4,5]$. Currently, there are three orally highly selective inhibitors of CDK4/6, namely palbociclib (PD0332991), ribociclib (LEE011) and abemaciclib (LY2835219).

CDK4/6 inhibitors have been extensively studied in various clinical trials for patients with HR+/HER2-negative MBC. In general, the eligible population in these trials may be classified as: a) sensitive to aromatase inhibitors (Al-sensitive), which included patients that are either naïve to Al or late relapsers (>12 months) since the stopping of the Al-based adjuvant treatment; or b) resistant to Al (Al-resistant), which included patients that are either pretreated with an Al in metastatic setting or relapsed during or early after ( $\leq 12$ months) the Al-based adjuvant treatment.

Palbociclib was the first inhibitor of CDK4/6 to receive an accelerated approval by Food and Drug Administration (FDA) in February 2015, in combination with letrozole as initial therapy for Al-sensitive advanced or metastatic BC, based on results from PALOMA-1 and PALOMA-2 trial [6-8]. More recently, based on data from PALOMA-3 trial, the indication was expanded to the combination with fulvestrant for both postmenopausal and pre-perimenopausal women with Al-resistant advanced or metastatic BC [9-11]. 
Ribociclib received FDA approval in March 2017, based on results from MONALEESA-2 trial, as initial endocrine-based therapy for Al-sensitive advanced or metastatic $\mathrm{BC}$, in combination with an aromatase inhibitor and then, based on results from MONALEESA-7 trial, it was also approved for the treatment of peri/premenopausal women [12-16]. Furthermore, based on the results of the MONALEESA-3 trial [17], ribociclib was also approved in combination with fulvestrant for postmenopausal women with both Al-sensitive and Al-resistant advanced or metastatic BC.

In September 2017, results from MONARCH-2 trial [18] lead to quick approval of abemaciclib in combination with fulvestrant in women Al-resistant advanced or metastatic BC. In addition, MONARCH-3 study showed that the addition of abemaciclib to non-steroidal aromatase inhibitor (NSAI) significantly improves progression free survival (PFS) in patients with Al-sensitive advanced or metastatic BC $[19,20]$. Abemaciclib has also been approved as a monotherapy for patients with HRpositive/HER2-negative MBC who have previously received endocrine therapy (ET) and chemotherapy, based on results from MONARCH-1 trial [21].

The inclusion of CDK4/6 inhibitors in combination with ET in international treatment guidelines, both for Al-sensitive and Alresistant patients, represents the most relevant advance in the management of HR-positive/HER2-negative advanced or metastatic BC over the last years [22]. Some controversies, however, still hold, particularly as to whether: 1. CDK4/6 inhibitors are effective or necessary for indolent, very late relapsing disease (i.e. those relapsing $>38$ months from stopping the adjuvant treatment); 2 . these drugs are effective in the most aggressive diseases (i.e. early relapse and/or with visceral metastases and/or with high tumour burden) or rather chemotherapy is indicated in these cases; 3 . there is different efficacy between different CDK4/6 inhibitors, particularly when the effect on overall survival (OS) is concerned. Indeed, only data from MONALEESA-3, MONALEESA-7 and MONARCH-2 trials show a significant OS improvement with adding CDK4/6 inhibitors to ET, while results from PALOMA-1, PALOMA-3 and MONALEESA-2 trials have not shown, so far, a statistically significant increase of OS for the combinations of palbociclib plus letrozole, palbociclib plus fulvestrant and ribociclib plus letrozole, respectively $[11,15,16,23-25]$.

We carried out a metanalysis of all randomized controlled trials (RCTs) with the aims of better characterising the efficacy of CDK4/6 inhibitors in some relevant subgroups of patients and of testing heterogeneity between different compounds with particular focus on their ability to improve OS.

\section{Materials And Methods}

This systematic review and meta-analysis was conducted using methods proposed by the Cochrane Collaboration, reported in accordance with the PRISMA statement (Supplemental Data) and was registered with PROSPERO [26, 27].

\subsection{Search strategy}

A comprehensive search of MEDLINE via PubMed and the Cochrane databases was performed using medical subject heading (MeSH) terms and text words related to CDK4/6 inhibitors and advanced or metastatic BC. Research was restricted from January 2010 until June 30, 2019 but two additional results, relevant to assess overall survival, were added:

MONALEESA-3 and MONARCH-2, presented at European Society for Medical Oncology Meeting (ESMO) in September 2019. A computerized search was also run in order to identify abstracts and presentations of relevant unpublished studies, reported at the Annual Meetings of the American Society of Clinical Oncology (ASCO), at the San Antonio Breast Cancer Symposium (SABC) and at ESMO meetings over the last three years. Additional studies were hand-searched on Clinicaltrials.gov. Relevant review articles and references from retrieved articles were screened for additional eligible studies. Screening of citation obtained from literature search was performed by two independent reviewers (MP and PC), as well as the assessment of studies eligibility. Any disagreement was discussed and resolved by consensus between both reviewers or consulting a third reviewer (MDL). A PRISMA flow diagram was prepared to document the process of studies selection.

\subsection{Eligibility criteria}


We included phase II and phase III RTCs which fulfil the following eligibility criteria: i) patients with HR-positive/HER2negative advanced or metastatic BC; ii) experimental arm including a selective inhibitor of CDK4/6 (palbociclib, ribociclib or abemaciclib); iii) control arm including standard of care (SOC) treatment +/- placebo); iv) availability of data regarding the primary outcome of interest (PFS/TTP reported in terms of hazard ratio and related Cls). SOC treatment includes ET only, such as aromatase inhibitors (i.e. anastrozole, letrozole, exemestane), oestrogen receptor modulators (i.e. tamoxifen) or selective oestrogen receptor downregulators (i.e. fulvestrant). Clinical trials that assessed the efficacy of CDK4/6 inhibitor as monotherapy were excluded, as well as clinical trials including chemotherapy based regimen and studies conducted in (neo)adjuvant setting. Cohort studies, case series, case reports and reviews were also excluded.

\subsection{Outcome for analysis}

The main outcome of interest was the investigator-assessed progression free survival (PFS), defined as the time from randomisation to objective disease progression or death for any reason. Secondary outcomes were overall survival (OS), defined as the time from randomisation to date of death due to any cause, and objective response rate (ORR; proportion of patients achieving a complete or partial response). For PFS and OS analysis, the treatment effect was expressed as hazard ratio (HR) of CDK4/6-ET arm over the ET arm, so that an HR greater than one comes out in favour of standard arm, while an HR less than one comes out in favour of experimental arm. For ORR a meta-analysis of single proportions has been implemented, in order to calculate an overall proportion. All estimates of treatment effect were accompanied by $95 \%$ confidence intervals.

\subsection{Data extraction and assessment of risk of bias}

Data from eligible studies were extracted using a custom-made spreadsheet and were checked for accuracy. The following information were extracted from each study: first author, publication year, ClinicalTrials.gov Identifier, study design, regimen details in both experimental and control arm, allocated patients for each arm, main patient's characteristics (median age, line of therapy, sensitivity to ET), HRs and ORRs for the whole study population and for major subgroups of interest. 95\% Cls related to HRs and ORRs were also extracted. Where available, the full protocol of each study was consulted to verify study objectives, population and other relevant information regarding study design and conduction. For publications reporting results from the same study, the most recent or complete publication reporting the information of interest was considered.

The risk of bias of included studies was assessed using the Cochrane Collaboration's tool; it is made up of six domains: sequence generation, allocation concealment, blinding, incomplete outcome data, selective outcome reporting and other potential bias [28]. Data extraction and risk of bias for each study was independently assessed by MP and PC and disagreements were discussed and resolved by consensus between both reviewers or consulting a third reviewer (MDL).

\subsection{Statistical methods}

The pooled estimates of HRs and overall proportions of objective responses, with two-sided $95 \%$ Cls, were computed for PFS, OS and ORR analysis, by using both a fixed-effect model according the inverse-variance method [29] and a random-effect model of DerSimonian and Laird [30], in order to obtain more appropriate estimates of the average treatment effect in case of between-study heterogeneity. The assumption of homogeneity between studies was tested with Cochran's Q statistics [31] and the measure of the degree of inconsistency across studies was assessed with Higgins'|^2 index [32]. Where available, predefined subgroup analyses were performed, in order to better understand if the treatment effect changes because of specific patients' characteristics. Results obtained from the analyses were displayed by generating a forest plot. To estimate the absolute gain in terms of PFS, meta-analytic survival curves were calculated as suggest by Parmar et al [33]. A sensitivity analysis was carried out by using the leave-one-out cross validation method that recalculates the pooled estimates omitting one study at a time; this analysis is able to capture whether some features of included studies influence the pooled estimates. Publication bias was assessed using funnel plots and regression tests, according to the method reported by Egger [34]. A Pvalue $<0.05$ was considered statistically significant. Data analysis was performed using R 3.4 .1 software packages [35, 36]. To estimate the absolute benefit in terms of PFS, pooled survival curves were generated by pooling the data of all trials, among Al-sensitive and Al-resistant patients. The number at risk and the total number of events were extracted from 
publications at specific time points based on available data, the survival probabilities were also extracted using the Digitizelt software (https://www.digitizeit.de/).

\section{Results}

\subsection{Study selection and characteristics}

The search strategy yielded 685 results from Pubmed, Cochrane database, conferences and clinicaltrials.gov. After the initial review of titles, 57 duplicates and further 593 results were discarded. We reviewed 35 abstract and 21 references were assessed for eligibility. Finally, we identified 8 randomized trials which fulfil the eligibility criteria. When possible, the latest publication of each trial was used for the meta-analysis. Two additional publications, presented at ESMO conference in September 2019, where included after database searching for OS analysis. The PRISMA flow diagram and the complete search strategy are available as Supplemental Data.

The eight RCTs included in this systematic review and meta-analysis were published between 2015 and 2019 and randomised a total of 4580 patients, of which 2802 received a CDK4/6 inhibitor (palbociclib, ribociclib or abemaciclib) in association with ET (NSAl, tamoxifene or fulvestrant) and 1778 received standard ET alone or in combination with placebo. Overall survival results from MONALEESA-3 and MONARCH-2 studies presented after database searching were also included for secondary outcome analysis $[23,24]$. The main characteristics of each trial included are summarised in Table 1. Palbociclib was tested in combination with letrozole $2.5 \mathrm{mg}$ /day in the PALOMA-1 and PALOMA-2 trial, for Al-sensitive patients, $[6-8,37]$ and in combination with fulvestrant $500 \mathrm{mg}$ every 28 days in the PALOMA-3 trial, for Al-resistant [11, 3840]. Ribociclib was investigated in combination with letrozole $2.5 \mathrm{mg} /$ day in the MONALEESA-2 trial for Al-sensitive postmenopausal women $[12,15,16,41-44]$, in combination with tamoxifene or NSAl (with goserelin to suppress ovarian function) in premenopausal Al-sensitive women in the MONALEESA-7 trial [16, 45], and in combination with fulvestrant both for Al-sensitive and Al-resistant patients in the MONALEESA-3 trial [17]. Finally, abemaciclib was used in combination with anastrozole $1 \mathrm{mg} /$ day or letrozole $2.5 \mathrm{mg} /$ day, as per physician choice, for Al-sensitive patients in the MONARCH-3 trial, [19, 20] and in combination with fulvestrant $500 \mathrm{mg}$ every 28 days for Al-resistant patients in the MONARCH-2 trial [18, 24]. The main outcome was the PFS for all trials and OS and ORR were secondary outcomes for all trials. OS estimates were available only for PALOMA-1, PALOMA-3, MONALEESA-2, MONALEESA-3, MONALEESA-7 and MONARCH-2 trials, while OS data were still pending for the PALOMA-2 and MONARCH-3 trials. 
Table 1

\begin{tabular}{|c|c|c|c|c|c|c|c|c|}
\hline $\begin{array}{l}\text { First author, } \\
\text { year (study } \\
\text { name) }\end{array}$ & Phase & Population & $\begin{array}{l}\text { Experimental } \\
\text { arm (n) }\end{array}$ & $\begin{array}{l}\text { Control arm } \\
\text { (n) }\end{array}$ & $\begin{array}{l}\text { Endocrine } \\
\text { status }\end{array}$ & $\begin{array}{l}\text { Median } \\
\text { PFS Exp } \\
\text { arm }\end{array}$ & $\begin{array}{l}\text { Median } \\
\text { PFS Ctrl } \\
\text { arm }\end{array}$ & $\begin{array}{l}\text { HR } \\
(95 \% \\
\text { Cl) }\end{array}$ \\
\hline $\begin{array}{l}\text { Hortobagyi } \\
\text { GN, } 2018 \\
\text { (MONALEESA- } \\
\text { 2) }\end{array}$ & III & $\begin{array}{l}\text { Post- } \\
\text { menopausal } \\
\text { Al-sensitive }\end{array}$ & $\begin{array}{l}\text { Ribociclib + } \\
\text { Letrozole } \\
\text { (334) }\end{array}$ & $\begin{array}{l}\text { Letrozole + } \\
\text { Placebo } \\
\text { (334) }\end{array}$ & Sensitive & $\begin{array}{l}25.3 \\
(23.0- \\
30.3)\end{array}$ & $\begin{array}{l}16 \\
(13.4- \\
18.2)\end{array}$ & $\begin{array}{l}0.568 \\
(0.457- \\
0.704)\end{array}$ \\
\hline $\begin{array}{l}\text { Slamon DJ, } \\
2018 \\
\text { (MONALEESA- } \\
\text { 3) }\end{array}$ & III & $\begin{array}{l}\text { Post- } \\
\text { menopausal } \\
\text { Al-sensitive } \\
\& \text { Al- } \\
\text { resistent }\end{array}$ & $\begin{array}{l}\text { Ribociclib + } \\
\text { Fulvestrant } \\
\text { (484) }\end{array}$ & $\begin{array}{l}\text { Fulvestrant } \\
\text { + Placebo } \\
\text { (242) }\end{array}$ & Mixed & $\begin{array}{l}20.5 \\
(18.5- \\
23.5)\end{array}$ & $\begin{array}{l}12.8 \\
(10.9- \\
16.3)\end{array}$ & $\begin{array}{l}0.593 \\
(0.480- \\
0.732)\end{array}$ \\
\hline $\begin{array}{l}\text { Tripathy D, } \\
2018 \\
\text { (MONALEESA- } \\
7 \text { ) }\end{array}$ & III & $\begin{array}{l}\text { Pre- } \\
\text { menopausal } \\
\text { Al-sensitive }\end{array}$ & $\begin{array}{l}\text { Ribociclib + } \\
\text { Tamoxifene } \\
\text { or NSAl+ } \\
\text { Goserelin } \\
\text { (335) }\end{array}$ & $\begin{array}{l}\text { Placebo + } \\
\text { Tamoxifene } \\
\text { or NSAl } \\
(337)\end{array}$ & Mixed & $\begin{array}{l}23.8 \\
(19.2- \\
\left.N R^{\star}\right)\end{array}$ & $\begin{array}{l}13.3 \\
(11.0- \\
16.4)\end{array}$ & $\begin{array}{l}0.553 \\
(0.441- \\
0.694)\end{array}$ \\
\hline $\begin{array}{l}\text { Sledge GW, } \\
2019 \\
\text { (MONARCH-2) }\end{array}$ & III & $\begin{array}{l}\text { Pre/Post- } \\
\text { menopausal } \\
\text { Al-resistant }\end{array}$ & $\begin{array}{l}\text { Abemaciclib } \\
+ \text { Fulvestrant } \\
\text { (446) }\end{array}$ & $\begin{array}{l}\text { Fulvestrant } \\
\text { + Placebo } \\
\text { (223) }\end{array}$ & Resistant & $\begin{array}{l}16.4 \text { (not } \\
\text { reported) }\end{array}$ & $\begin{array}{l}9.3 \text { (not } \\
\text { reported) }\end{array}$ & $\begin{array}{l}0.553 \\
(0.449- \\
0.681)\end{array}$ \\
\hline $\begin{array}{l}\text { Johnston S, } \\
2019 \\
\text { (MONARCH-3) }\end{array}$ & III & $\begin{array}{l}\text { Pre/Post- } \\
\text { menopausal } \\
\text { Al-sensitive }\end{array}$ & $\begin{array}{l}\text { Abemaciclib } \\
\text { + NSAI (328) }\end{array}$ & $\begin{array}{l}\text { Placebo + } \\
\text { NSAI (165) }\end{array}$ & Sensitive & $\begin{array}{l}28.1 \text { (not } \\
\text { reported) }\end{array}$ & $\begin{array}{l}14.7 \text { (not } \\
\text { reported) }\end{array}$ & $\begin{array}{l}0.540 \\
(0.418- \\
0.698)\end{array}$ \\
\hline $\begin{array}{l}\text { Finn RS, } 2015 \\
\text { (PALOMA-1) }\end{array}$ & II & $\begin{array}{l}\text { Pre/Post- } \\
\text { menopausal } \\
\text { Al-sensitive }\end{array}$ & $\begin{array}{l}\text { Palbociclib } \\
\text { + Letrozole } \\
\text { (84) }\end{array}$ & $\begin{array}{l}\text { Letrozole } \\
(81)\end{array}$ & Sensitive & $\begin{array}{l}20.2 \\
(13.8- \\
27.5)\end{array}$ & $\begin{array}{l}10.2 \\
(5.7- \\
12.6)\end{array}$ & $\begin{array}{l}0.488 \\
(0.319- \\
0.748)\end{array}$ \\
\hline $\begin{array}{l}\text { Rugo HS, } \\
2019 \\
\text { (PALOMA-2) }\end{array}$ & III & $\begin{array}{l}\text { Pre/Post- } \\
\text { menopausal } \\
\text { Al-sensitive }\end{array}$ & $\begin{array}{l}\text { Palbociclib } \\
\text { + Letrozole } \\
\text { (444) }\end{array}$ & $\begin{array}{l}\text { Letrozole } \\
(222)\end{array}$ & Sensitive & $\begin{array}{l}27.6 \\
(22.4- \\
30.3)\end{array}$ & $\begin{array}{l}14.5 \\
(12.3-17- \\
1)\end{array}$ & $\begin{array}{l}0.563 \\
(0.461- \\
0.687)\end{array}$ \\
\hline $\begin{array}{l}\text { Cristofanilli M, } \\
2016 \\
\text { (PALOMA-3) }\end{array}$ & III & $\begin{array}{l}\text { Pre/Post- } \\
\text { menopausal } \\
\text { Al-resistant }\end{array}$ & $\begin{array}{l}\text { Palbociclib } \\
\text { + Fulvestrant } \\
\text { (521) }\end{array}$ & $\begin{array}{l}\text { Fulvestrant } \\
+ \text { Placebo } \\
(347)\end{array}$ & Resistant & $\begin{array}{l}9.5(9.2- \\
11.0)\end{array}$ & $\begin{array}{l}4.6(3.5- \\
5.6)\end{array}$ & $\begin{array}{l}0.46 \\
(0.36- \\
0.59)\end{array}$ \\
\hline
\end{tabular}

\subsection{Risk of bias}

Overall the risk of selection, performance, attrition, detection and reporting bias was very low, because all trials were double blind, with exception of PALOMA-1 study that was a phase II, open-label study. The risk-of-bias in each study is reported as Supplemental Data.

\subsection{Progression free survival}

PFS hazard ratios were directly available for all included studies. Single study HRs ranged from 0.46 to 0.59 and were all statistically significant. Pooled analysis showed a statistically significant improvement in PFS for patients treated with CDK4/6 inhibitor in combination with ET versus patients treated with ET alone (HR 0.547 [95\% Cl 0.504, 0.594], p-value< 0.0001). Both a fixed-effect model and a random-effect model were implemented, as initially planned, also if no heterogeneity between studies was detected ( $\left.\right|^{\wedge} 20 \%$; chi^2 2.95, p 0.89), as well as publication bias (Egger test, p 0.09). Forest plot, test for publication bias detection and sensitivity analysis are available as Supplemental data.

\subsection{Subgroups analysis}

A subgroup analysis was implemented according the following criteria: endocrine sensitivity, site of metastasis, number of organs involved and treatment free interval. 
Based on the aforementioned definitions, we pooled PFS estimates for Al-sensitive patients and Al-resistant patients among all trials included in this analysis. Patients with 'de novo' disease were considered as a separate group. A total of 5329 patients were included in this group, of which 2852 were Al-sensitive, 1536 Al-resistant and 941 patients had de novo disease. MONALEESA-2, MONALEESA-7, MONARCH-3, PALOMA-1 and PALOMA-2 studies enrolled exclusively Al-sensitive patients while MONARCH-2 and PALOMA-3 enrolled Al-resistant patients only; MONALEESA-3 enrolled both sensitive and resistant patients. It also enrolled 'de novo' patients (19\% of the total), but separate estimates for these patients are not available. 'De novo' patients of the MONALEESA-3 trial were, therefore, included in the Al-sensitive for the purpose of this meta-analysis. No between-group difference was observed (I^2 0\%; chi^2 4.92, p-value 0.960). The pooled HRs were very similar in Al-sensitive, 'de novo' Al-resistant patients.

Treatment free interval (TFI) was defined as the time from the end of the adjuvant therapy to randomization. TFI was analysed at four time points: $<=24$ months, $>24$ months, $<=36$ months and $>36$ months. Overall 1391 patients were included in this analysis, of which 199 had a TFI < = 24 months, 686 had a TFI $>24$ months, 244 had a TFI $<=36$ months and 262 had a TFI > 36 months. No significant heterogeneity was observed (I^2 0\%; chi^2 6.22, p-value 0.622). The pooled analysis confirm the beneficial effect of adding CDK 4/6 inhibitor to standard ET regardless the treatment free interval. The estimated pooled HRs according the Al-sensitivity and TFI is shown in Fig. 1.

Site of Metastases and number of metastatic sites

A total of 5862 patients were grouped by site of metastasis, of which 2429 had visceral disease (including patients belonging to liver-or-lung subgroup from MONALEESA-2, MONALEESA-3 and MONALEESA-7 trials), 929 had bone only disease and 2504 had no bone only disease (including patients with both visceral and bone disease). The a total of 2845 patients was grouped by number of metastatic sites, of which 782 had only one metastatic site at study entry, 635 had two metastatic sites and 1428 had three or more metastatic sites. No heterogeneity was detected, so the fixed effect model was considered. The pooled results of meta-analysis, showed a statistically significant improvement in PFS with a similar HR for all these subgroups (Fig. 2).

\subsection{Objective Response}

ORR data were available for all studies. Pooled estimates of ORR were summarized as bar-plot, forest plots are available as Supplemental Data. Figure 3 shows the bar-plot of pooled ORR in all randomly assigned patients and in patients with measurable disease according Al-sensitivity. Overall 2318 patients treated with CDK 4/6 inhibitor plus ET and 1536 patients treated with ET alone were included in this analysis, while 1781 patients had measurable disease (1195 treated with CDK 4/6 inhibitor + ET and 586 treated with ET alone). The meta-analysis shows an increased ORR in patients treated with CDK 4/6 inhibitors, both in Al-sensitive (pooled ORR $=43.3 \%$ for CDK4/6 inhibitors treated patients) and Al-resistant group (pooled ORR $=26.5 \%$ for CDK4/ 6 inhibitor treated patients). Patients treated with CDK $4 / 6$ inhibitor reached a pooled ORR of $55 \%$ in Al-sensitive group and 35.6\% Al-resistant group. Results of analysis according the CDK 4/6 inhibitor are available as Supplemental Data.

\subsection{Overall survival}

Overall survival (OS) data were available only for MONALEESA-2, MONALEESA-3, MONALEESA-7, MONARCH-2, PALOMA-1 and PALOMA-3 trial $[11,15,16,23-25]$. This analysis included a total of 3421 patients, of which 2030 treated with CDK $4 / 6$ inhibitors and 1391 treated with ET alone. The pooled HR indicates a statistically significant reduction in the risk of dying for patients receiving the $\mathrm{CDK} 4 / 6$ inhibitor (HR 0.763 [95\% $\mathrm{Cl} 0.683 ; 0.852]$, p-value < 0.0001 ); this effect is independent of whether patients were Al-sensitive or not. When grouped by CDK4/6 inhibitor, a statically significant reduction in the hazard of dying was apparent for Ribociclib and Abemaciclib only, but not for Palbociclib (Fig. 4). However, the test for heterogeneity 
(I^2 0\%; chi^2 1.44, p-value 0.919) suggests that discrepant OS results among different CDK4/6 inhibitors may be explained by chance. Meta-analysis of OS in overall population is available as Supplemental Data.

\subsection{Pooled survival curves}

Since no heterogeneity emerged from the overall PFS analysis (A), it is possible to pool the PFS data to better estimate the absolute benefit gained by adding a CDK4/6 inhibitor to ET. Pooled PFS curves show a median PFS of 26.5 months in Alsensitive patients treated with CDK 4/6 inhibitors (10.9 months improvement over ET alone). In the Al-resistant population, median PFS was 14.1 months for patients treated with CDK 4/6 inhibitors (7 months improvement over ET alone) (Fig. 5).

\section{Discussion}

The development of CDK 4/6 inhibitors has changed the therapeutic management of HR + MBC. Palbociclib, ribociclib, and abemaciclib are all orally active, highly selective reversible inhibitors of CDK4 and CDK6 approved by the Food and Drug Administration, the European Medicine Agency (EMA) and other regulatory agencies worldwide for HR + MBC in combination with Als and fulvestrant. While there is general agreement on the efficacy and the manageable toxicity of these drugs, some controversies still hold in the interpretation of clinical trials data, particularly when subgroups data are concerned. This metanalysis was carried out to contribute to resolve controversial issues by: 1. providing more reliable estimates of efficacy in some controversial subgroups; 2 . increasing the statistical power to evaluate the impact on OS; 3 . testing for significant heterogeneity between different compounds; 4 . refining the overall estimates of efficacy, in case of no heterogeneity detection.

Despite uniform recommendation from guidelines, chemotherapy is still overused in $\mathrm{HR}+\mathrm{MBC}$, particularly in situations in which an intense tumour debulking is desirable. This behaviour relies on the diffused perception, among both oncologists and patients, that chemotherapy is a more potent treatment, yielding on average a higher ORR than ET-based treatments. In contrast, our meta-analysis confirms that the combination of a CDK4/6 inhibitors and ET yields a very high rate of tumour regression, which is on average as high as $55 \%$ for patients with measurable disease, with no heterogeneity among different compounds. To the best of our knowledge, this ORR is higher than we would expect with mono-chemotherapy and comparable (but still lower) to what we would expect with an aggressive polychemotherapy in HR + pts populations. Therefore, the use of chemotherapy as the best mean to obtain tumour debulking in HR + MBC should be definitely considered obsolete.

It has been argued that the addition of CDK4/6 inhibitors to ET may not produce relevant benefits for less aggressive tumours, namely tumours with bone-only metastases or long TFI or limited number of metastases. This debate was based on subgroup analyses from single trials. In contrast, our meta-analysis demonstrates that adding a CDK4/6 inhibitors to ET is beneficial in terms of PFS irrespective of the presence or not of visceral metastases, the number of metastatic sites, and the length of the TFI. The test for heterogeneity in these subgroup analysis indicates that minor differences, if present, are due to chance; we should, therefore, assume that the pooled estimate in the overall population is the best estimate of the treatment effect size in each subgroup of patients.

Overall survival data are relatively immature. Despite this, pooling current estimates from relevant trials demonstrates that the addition of CDK4/6 inhibitors to ET does produce an OS improvement. This improvement is evident both in Al-sensitive pts and Al-resistant pts, strongly supporting the use of CDK4/6 inhibitors as gold standard treatment in both patient populations. A controversial issue is whether these drugs are equally effective in prolonging OS, because, so far, only Ribociclib and Abemaciclib have demonstrated a statistically significant improvement of OS in at least one trial [24]. In our meta-analysis, when pooling data from different trials for each single drug, Palbociclib still remains the only class member to not show a statistically significant HR for OS. However, this result should be interpreted with caution because the test for interaction indicates that the differences between the pooled HRs for the three CDK4/6 inhibitors may well be ascribed to chance (Fig. 4). Therefore, as above, one should assume that the best HR estimate for each drug is that of the overall pooled estimate (HR = 
$0.76 ; p<0.001$ ) (Fig. 4). Yet, because such results pertain to indirect comparisons, we cannot exclude that there could still be moderate, but clinically relevant, differences in efficacy between different compounds, which could only be identified in a direct randomized comparison.

Some limitation of our study need discussion. First, like for all meta-analysis of published data a publication bias may in theory have overemphasized a positive result. However, we used data from trials published in extenso and from trials reported at meetings to minimize publication bias and the results of the egger test indicates the absence of a relevant publication bias in our analysis. Second, akin all studies based on aggregated data, our meta-analysis, does not reach the level of evidence obtainable with a meta-analysis based on individual patient data (IPD) because: (1) it is impossible to determine the appropriateness of random assignment procedures; (2) trial heterogeneity can only be statistically tested, but never verified; and (3) it is not possible to do an intention-to-treat analysis because data from excluded patients cannot be retrieved. However, in our case, all authors declared their data were based on the intention-to-treat principle and the analysis of potential biases indicates that major biases in the included trials are unlikely. Furthermore, provided a rigorous methodology is used, pooling aggregated data, as in our case, yields information that is far superior to the simple descriptive across trial comparison.

\section{Conclusions}

Our meta-analysis confirms the efficacy of CDK4/6 inhibitors overall and in major patients subgroups, helps highlighting differences and similarities between different compounds and provides pooled (more precise) estimates of the effect size for PFS, OS and ORR. These results lend further strength to the evidence from single RCTs, supporting the use of CDK4/6 inhibitors in combination with ET as standard treatment for most HR + MBC pts.

\section{Declarations}

Ethical Approval and Consent to participate: Not Applicable

Consent for publication: Not Applicable

Availability of supporting data: The datasets generated during the current meta-analysis is available from the corresponding author on reasonable request. All data analysed during this meta-analysis are included in the corresponding published articles, as reported in table 1.

Competing interests: The authors declare that they have no competing interests

Funding: None

Authors' contributions: Study concepts and study design were developed by MP, MDL and PC. MP, PC and GDG were responsible for data acquisition and quality control of data and algorithms. Data analysis and its interpretation were perfomed by MP, PC, MR and SC. MP and PC performed all statistical analysis. MP, MDL and DC were responsible for manuscript preparation and editing. MDL, MP, PC, FN, GI, RC, VDL, FDR contributed in manuscript review. All authors read and approved the final manuscript.

Acknowledgements: Not Applicable

\section{References}

1. Siegel RL, Miller KD, Jemal A. Cancer statistics, 2018. CA Cancer J Clin 68: 7-30, 2018

2. Cancer Genome Atlas Network: Comprehensive molecular portraits of human breast cancer. Nature 490:61-70, 2012

Page $10 / 19$ 
3. Zardavas D, Baselga J, Piccart M: Emerging targeted agents in metastatic breast cancer. Nat Rev Clin Oncol 10:191-210, 2013

4. O'Leary B, Finn RS, Turner NC. Treating cancer with selective CDK4/6 inhibitors. Nat Rev Clin Oncol 13: 417-30, 2016

5. Finn RS, Dering J, Conklin D, et al. PD 0332991, a selective cyclin D kinase 4/6 inhibitor, preferentially inhibits proliferation of luminal estrogen receptor-positive human breast cancer cell lines in vitro. Breast Cancer Res 11: R77, 2009

6. Finn RS, Crown JP, Lang I, et al: The cyclin-dependent kinase 4/6 inhibitor palbociclib in combination with letrozole versus letrozole alone as first-line treatment of estrogen receptor-positive, HER2-negative, advanced breast cancer (PALOMA-1/TRIO-18): a randomised phase 2 study. Lancet Oncol 16:25e35, 2015

7. Finn RS, Martin M, Rugo HS, et al: Palbociclib and Letrozole in Advanced Breast Cancer. N Engl J Med. Nov 17; 375(20):1925-1936, 2016

8. Rugo HS, Finn RS, Diéras V, et al: Palbociclib plus letrozole as first-line therapy in estrogen receptor-positive/human epidermal growth factor receptor 2-negative advanced breast cancer with extended follow-up. Breast Cancer Res Treat 174(3):719-729, 2019

9. Turner NC, Ro J, André F, et al: Palbociclib in Hormone-Receptor-Positive Advanced Breast Cancer. N Engl J Med 373 (3), 209-19, 2015

10. Verma S, Bartlett CH, Schnell P, et al: Palbociclib in Combination With Fulvestrant in Women With Hormone ReceptorPositive/HER2-Negative Advanced Metastatic Breast Cancer: Detailed Safety Analysis From a Multicenter, Randomized, Placebo-Controlled, Phase III Study (PALOMA-3). Oncologist 21(10):1165-1175, 2016

11. Turner NC, Slamon DJ, Ro J, Bondarenko I, et al: Overall Survival with Palbociclib and Fulvestrant in Advanced Breast Cancer. N Engl J Med 379(20):1926-1936, 2018

12. Hortobagyi GN, Stemmer SM, Burris HA, et al: Ribociclib as First-Line Therapy for HR-Positive, Advanced Breast Cancer. N Engl J Med Nov 3;375(18):1738-1748, 2016

13. Hortobagyi GN, Stemmer SM, Burris HA, et al: Updated results from MONALEESA-2, a phase 3 trial of first-line ribociclib + letrozole in hormone receptor-positive (HR+), HER2-negative (HER2), advanced breast cancer (ABC). Journal of Clinical Oncology 35:15_suppl, 1038-1038, 2017

14. Syed YY. Ribociclib: First Global Approval. Drugs 77(7):799-807, 2017

15. Hortobagyi GN, Stemmer SM, Burris HA, et al: Updated results from MONALEESA-2, a phase III trial of first-line ribociclib plus letrozole versus placebo plus letrozole in hormone receptor-positive, HER2-negative advanced breast cancer. Ann Oncol Jul 1;29(7):1541-1547, 2018

16. Im SA, Lu YS, Bardia A, et al: Overall Survival with Ribociclib plus Endocrine Therapy in Breast Cancer. N Engl J Med 381 (4), 307-316, 2019

17. Slamon DJ, Neven P, Chia S, et al: Phase III Randomized Study of Ribociclib and Fulvestrant in Hormone ReceptorPositive, Human Epidermal Growth Factor Receptor 2-Negative Advanced Breast Cancer: MONALEESA-3. J Clin Oncol 36 (24), 2465-2472, 2018

18. Sledge GW, Toi M, Neven P, et al. MONARCH 2: abemaciclib in combination with fulvestrant in patients with HR+/HER2advanced breast cancer who progressed on endocrine therapy. J Clin Oncol 35, 2017 (suppl; abstr 1000).

19. Goetz MP, Toi M, Campone M, et al: MONARCH 3: Abemaciclib As Initial Therapy for Advanced Breast Cancer. J Clin Oncol Nov 10;35(32):3638-3646, 2017

20. Johnston S, Martin M, Di Leo A, et al: MONARCH 3 final PFS: a randomized study of abemaciclib as initial therapy for advanced breast cancer. NPJ Breast Cancer 17; 5:5, 2019

21. Dickler MN, Tolaney SM, Rugo HS, et al: MONARCH1: Results from a phase Il study of abemaciclib, a CDK4 and CDK6 inhibitor, as monotherapy, in patients with HR+/HER2- breast cancer, after chemotherapy for advanced disease. J Clin Oncol 34, 2016 (suppl; abstr 510) 
22. Rugo HS, Rumble RB, Macrae E, et al: Endocrine Therapy for Hormone Receptor-Positive Metastatic Breast Cancer: American Society of Clinical Oncology Guideline. J Clin Oncol 34(25):3069-103, 2016

23. Slamon DJ, Neven P, Chia S, et al: Overall survival results from the phase II MONALEESA-3 study of fulvestrant +/ribociclib in postmenopausal patients with HR+/HER2- advanced breast cancer. Presented at European Society of Medical Oncology Congress, Barcelona, Spain, 27 Sep - 01 Oct 2019

24. Sledge GW Jr, Toi M, Neven P, et al: The Effect of Abemaciclib Plus Fulvestrant on Overall Survival in Hormone ReceptorPositive, ERBB2-Negative Breast Cancer That Progressed on Endocrine Therapy-MONARCH 2: A Randomized Clinical Trial. JAMA Oncol September 29, 2019

25. Finn RS, Crown J, Lang I, et al: Overall survival results from the randomized phase Il study of palbociclib (P) in combination with letrozole $(\mathrm{L})$ vs letrozole alone for frontline treatment of ER+/HER2- advanced breast cancer (PALOMA1; TRIO-18). J Clin Oncol 35:1001-1001, 2017

26. Higgins JPT, Green S (editors). Cochrane Handbook for Systematic Reviews of Interventions Version 5.1.0. The Cochrane Collaboration, 2011.

27. Liberati A, Altman DG, Tetzlaff J, et al: The PRISMA statement for reporting systematic reviews and meta-analyses of studies that evaluate health care interventions: explanation and elaboration. J Clin Epidemiol 62(10):e1-34, 2009

28. Higgins JPT, Altman DG. Chapter 8: Assessing risk of bias in include studies. In: Higgins JPT, Green S, eds. Cochrane Handbook for Systematic Reviews of Interventions Version 5.1.0. The Cochrane Collaboration, 2011.

29. Deeks JJ, Altman DG, Bradburn MJ: Systematic reviews in health care: meta-analysis in context. Cap. 15 - John Wiley \& Sons, 2008

30. DerSimonian R and Laird N: Meta-Analysis in Clinical Trials. Controlled Clinical Trials, 7, 177-188, 1986

31. Cochran WG: The combination of estimates from different experiments. Biometrics 10:101-129, 1954

32. Higgins JPT, Thompson SG, Deeks JJ, et al: Measuring inconsistency in meta-analyses. BMJ Sep 6; 327(7414): 557560,2003

33. Parmar MK, Torri V, Stewart L: Extracting summary statistics to perform meta-analyses of the published literature for survival endpoints. Stat Med 17:2815-2834, 1998

34. Egger M, Davey Smith G, Schneider M, et al: Bias in meta-analysis detected by a simple, graphical test. BMJ 315:629634, 1997

35. Schwarzer G. General Package for Meta-Analysis. 2017. https://github.com/guido-s/meta

36. Viechtbauer, W: Conducting meta-analyses in R with the metafor package. Journal of Statistical Software, 36(3), 1-48, 2010

37. Finn RS, Crown JP, Ettl J, et al: Efficacy and safety of palbociclib in combination with letrozole as first-line treatment of ER-positive, HER2-negative, advanced breast cancer: expanded analyses of subgroups from the randomized pivotal trial PALOMA-1/TRIO-18. Breast Cancer Res Jun 28; 18(1):67, 2016

38. Cristofanilli M, Turner NC, Bondarenko I, et al: Fulvestrant plus palbociclib versus fulvestrant plus placebo for treatment of hormone-receptor-positive, HER2-negative metastatic breast cancer that progressed on previous endocrine therapy (PALOMA-3): final analysis of the multicentre, double-blind, phase 3 randomised controlled trial. Lancet Oncol Apr;17(4):425-39, 2016

39. Iwata H, Im SA, Masuda N, et al: PALOMA-3: Phase III Trial of Fulvestrant With or Without Palbociclib in Premenopausal and Postmenopausal Women With Hormone Receptor-Positive, Human Epidermal Growth Factor Receptor 2-Negative Metastatic Breast Cancer That Progressed on Prior Endocrine Therapy-Safety and Efficacy in Asian Patients. J Glob Oncol 3(4):289-303, 2017

40. Loibl S, Turner NC, Ro J, et al: Palbociclib Combined with Fulvestrant in Premenopausal Women with Advanced Breast Cancer and Prior Progression on Endocrine Therapy: PALOMA-3 Results. Oncologist 22(9):1028-1038, 2017 
41. Janni W, Alba E, Bachelot T, et al: First-line ribociclib plus letrozole in postmenopausal women with HR+, HER2-advanced breast cancer: Tumor response and pain reduction in the phase 3 MONALEESA-2 trial. Breast Cancer Res Treat 169 (3), 469-479, 2018

42. Sonke GS, Hart LL, Campone M, et al: Ribociclib with letrozole vs letrozole alone in elderly patients with hormone receptor-positive, HER2-negative breast cancer in the randomized MONALEESA-2 trial. Breast Cancer Res Treat 167(3):659-669, 2018

43. Blackwell KL, Paluch-Shimon S, Campone M, et al: Subsequent treatment for postmenopausal women with hormone receptor-positive, HER2-negative advanced breast cancer who received ribociclib + letrozole vs placebo + letrozole in the phase III MONALEESA-2 study. Presented at San Antonio Breast Cancer Symposium, San Antonio, Texas, December 5-9, 2017 (P5-21-18)

44. O'Shaughnessy J, Petrakova K, Sonke GS, et al: Ribociclib plus letrozole versus letrozole alone in patients with de novo HR+, HER2- advanced breast cancer in the randomized MONALEESA-2 trial. Breast Cancer Res Treat 168(1):127-134, 2018

45. Tripathy D, Im SA, Colleoni M, et al: Ribociclib plus endocrine therapy for premenopausal women with hormone-receptorpositive, advanced breast cancer (MONALEESA-7): a randomised phase 3 trial. Lancet Oncol S1470-2045(18)30292-4, 2018

\section{Additional File}

Additional file contains supplemental data such as full search strategy, the risk of bias graph, the funnel plot for publication bias detection, the sensitivity analysis performed and the forest plot of overall progression free survival.

\section{List Of Abbreviations}




\begin{tabular}{|ll|}
\hline Abbreviation & Definition \\
\hline Als & Aromatase Inhibitors \\
\hline BC & Breast Cancer \\
\hline CDK 4/6 & Cyclin Dependent Kinase 4/6 \\
\hline Cls & Confidence Intervals \\
\hline EMA & European Medicine Agency \\
\hline ET & Endocrine Therapy \\
\hline FDA & Food and Drug Administration \\
\hline HR+ & Hormone Receptor Positive \\
\hline IPD & Individual Patients data \\
\hline MBC & Metastatic Breast Cancer \\
\hline NSAl & Non Steroidal Aromatase Inhibitors \\
\hline ORR & Objective Response Rate \\
\hline OS & Overall Survival \\
\hline PFS & Progression Free Survival \\
\hline SOC & Standard Of Care \\
\hline TFI & Treatment Free Interval \\
\hline TTP & Time To Progression \\
\hline
\end{tabular}

Figures 


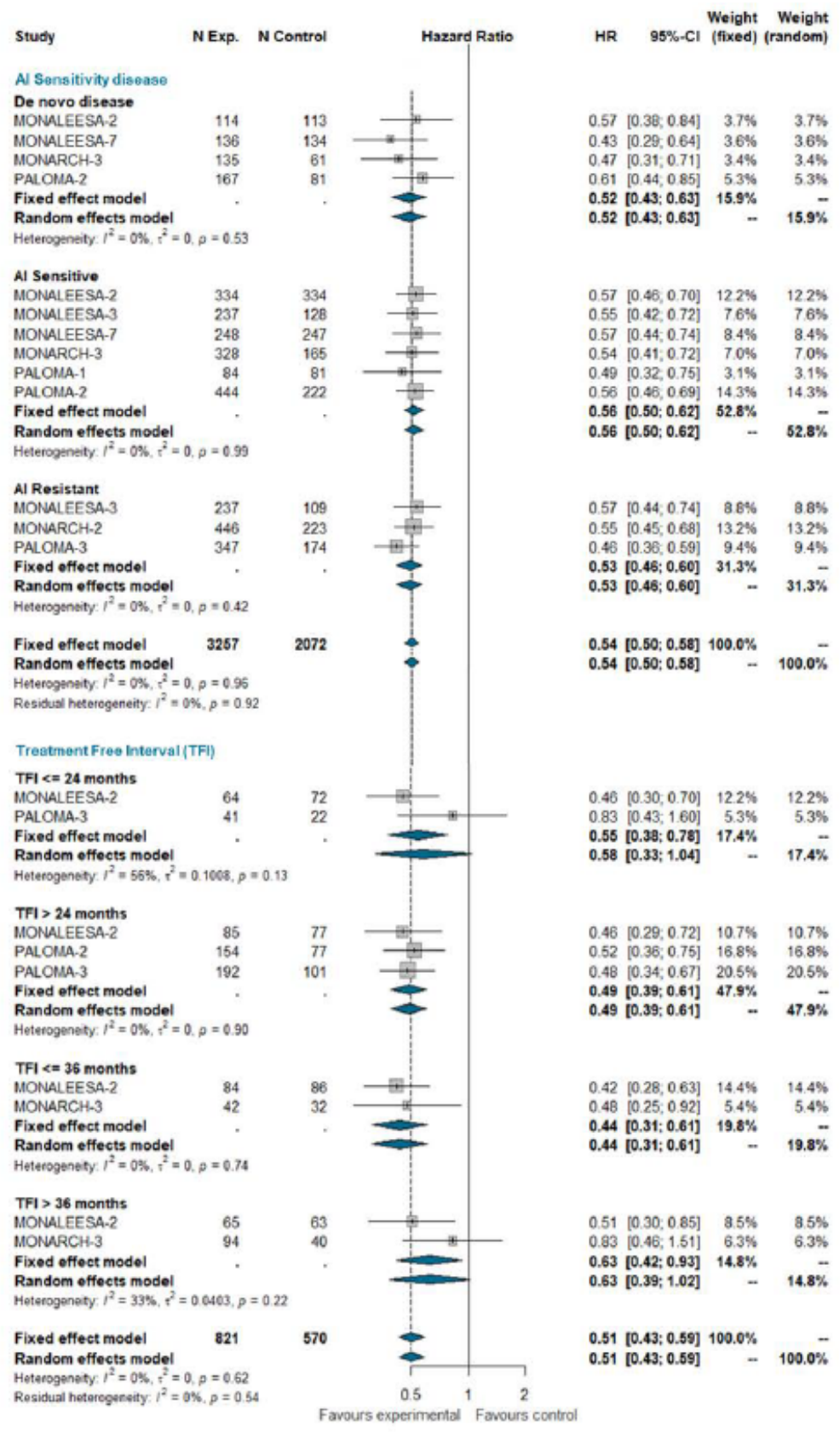

\section{Figure 1}

Pooled comparison of PFS according endocrine sensitivity and treatment free interval. 


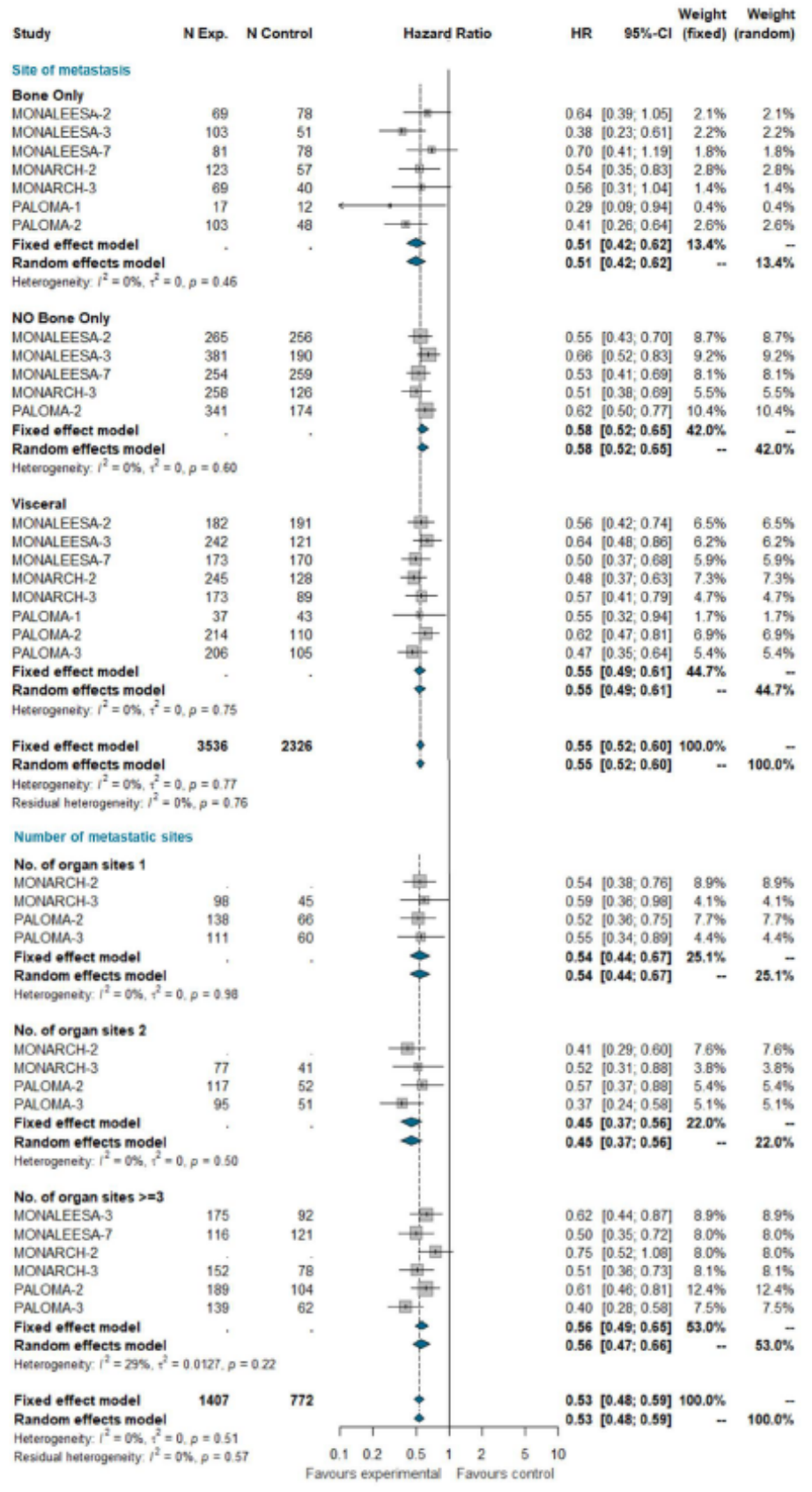

Figure 2

Pooled comparison of PFS according site of metastasis (visceral disease, bone only disease and no bone only disease) and number of organs involved $(1,2,3+)$. 


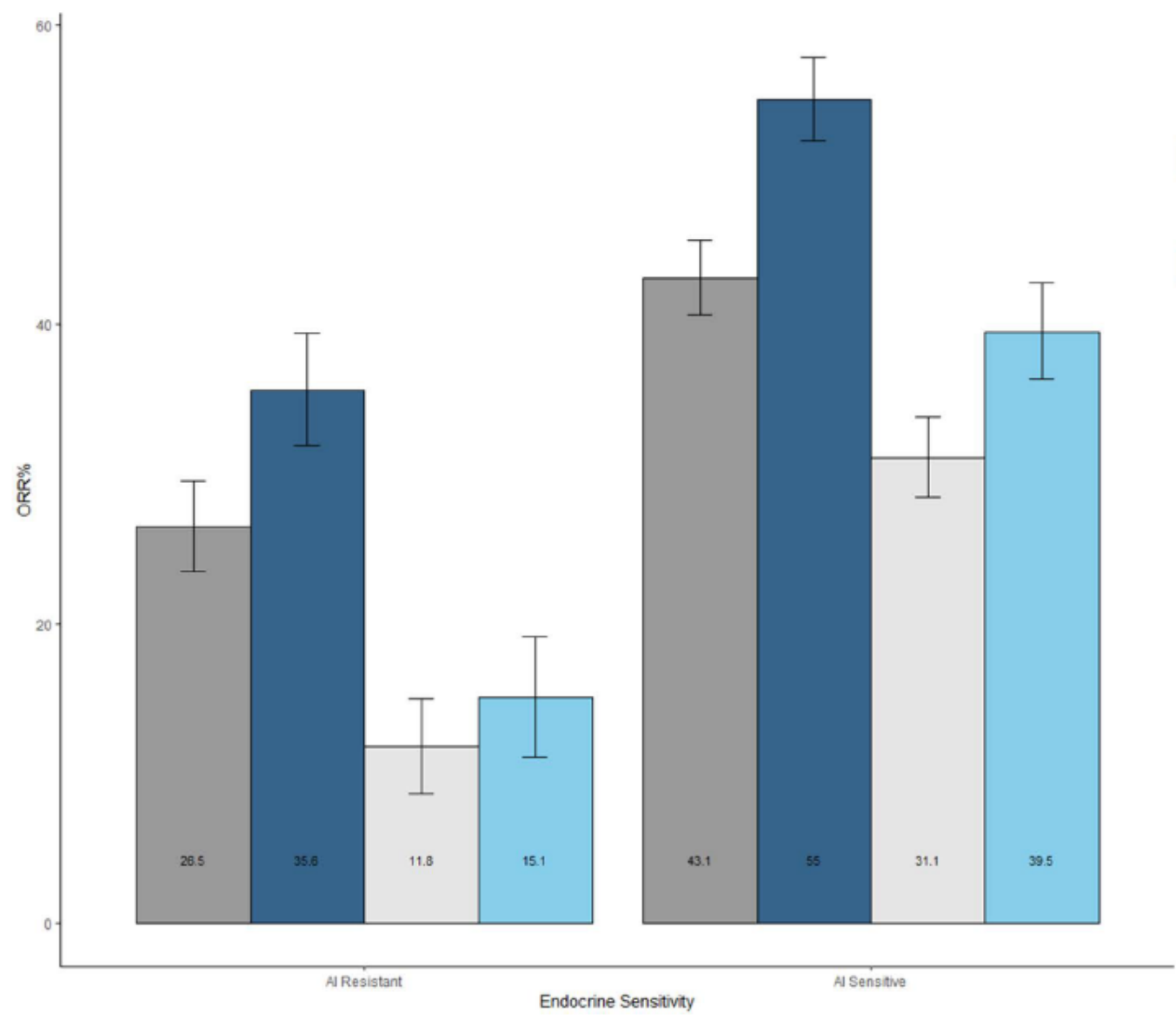

Treatment Group

OT + CDK in all randomized pts

OT + CDK in pts with measurable disease

OT alone in all randomized pts

OT alone in pts with measurable disease

\section{Figure 3}

Bar-plot of pooled ORR in all randomly assigned patients and in patients with measurable disease according ET sensitivity status. 


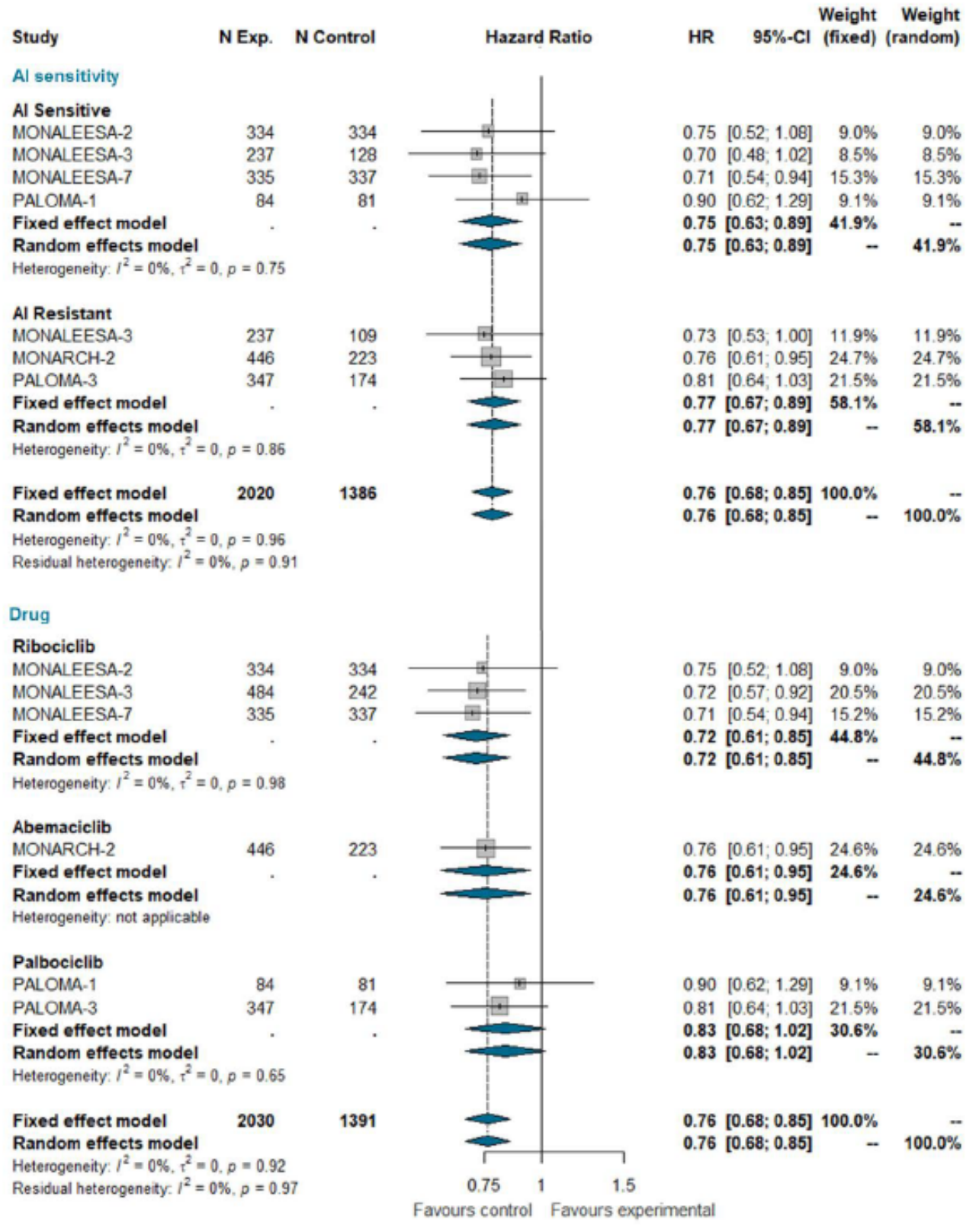

\section{Figure 4}

Meta-analysis of overall survival (OS) grouped by Al sensitivity and CDK 4/6 inhibitor. 


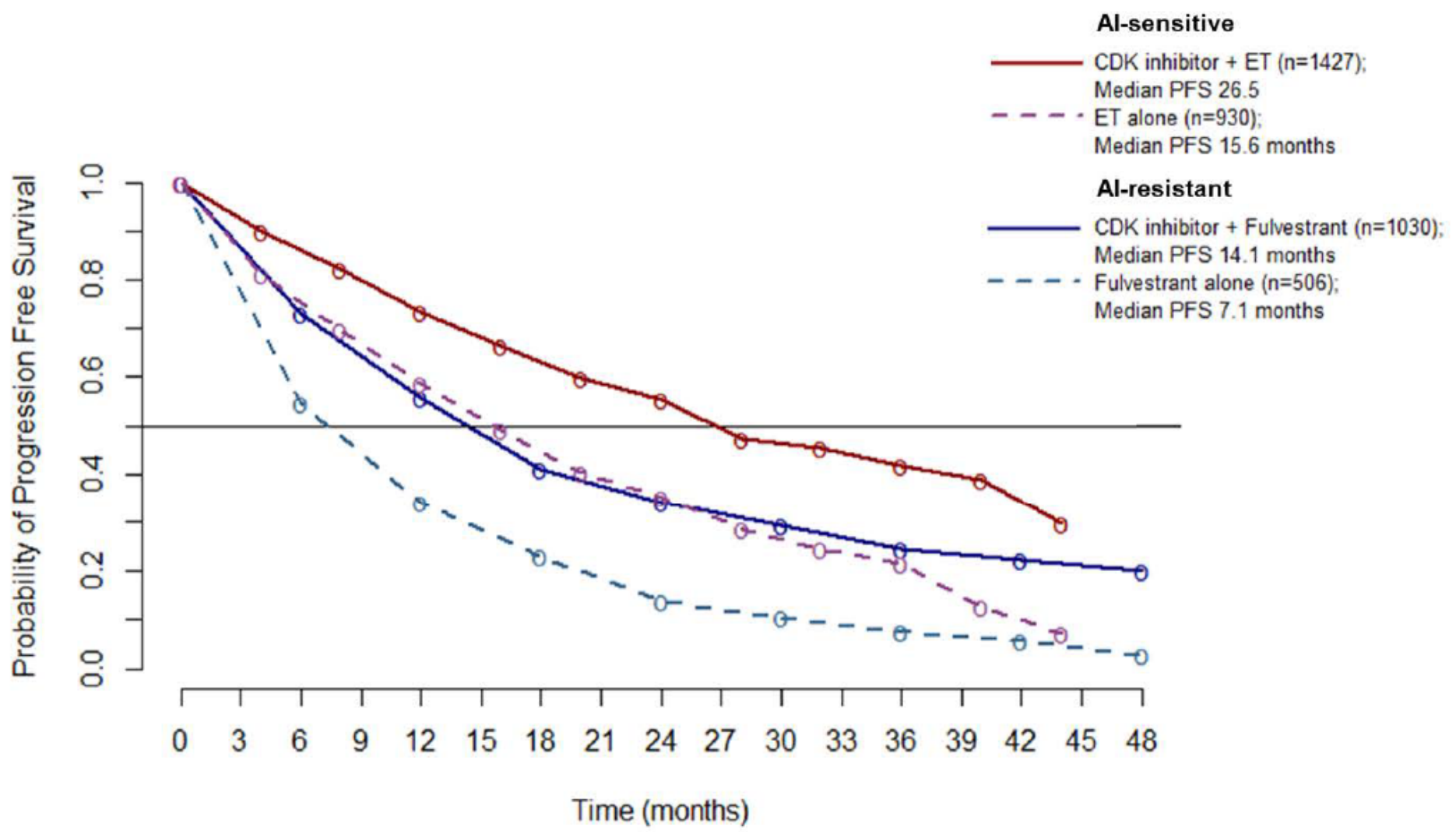

Figure 5

Meta-curves of PFS for Al-sensitive and Al-resistant patients.

\section{Supplementary Files}

This is a list of supplementary files associated with this preprint. Click to download.

- AdditionaFile1.docx 